\title{
Interferon- $\gamma$ mRNA attenuates its own translation by activating PKR: A molecular basis for the therapeutic effect of interferon- $\beta$ in multiple sclerosis
}

\author{
Raymond Kaempfer ${ }^{1}$ \\ ${ }^{1}$ Department of Molecular Virology, The Hebrew University-Hadassah Medical School, 91120 Jerusalem, Israel
}

PKR, the interferon (IFN)-inducible protein kinase activated by double-stranded RNA, inhibits translation by phosphorylating the initiation factor eIF2 $\alpha$ chain. Uniquely, human IFN- $\gamma$ mRNA uses local activation of PKR in the cell to control its own translation yield. IFN- $\gamma$ mRNA activates PKR through a structure in its 5'- region harboring a pseudoknot which is critical for PKR activation. Mutations that impair pseudoknot stability reduce the ability of IFN- $\gamma$ mRNA to activate PKR and strongly increase its translation efficiency. The cis-acting RNA element in IFN- $\gamma$ mRNA functions as a biological sensor of intracellular PKR levels. During an immune response, as IFN- $\gamma$ and other inflammatory cytokines build up in the cell's microenvironment, they act to induce higher levels of PKR in the cell, resulting in a more extensive activation of PKR by IFN- $\gamma$ mRNA. With the resulting phosphorylation of eIF2 $\alpha$, a negative feedback loop is created and the production of IFN- $\gamma$ is progressively attenuated. We propose that the therapeutic effect of IFN- $\beta$ in multiple sclerosis may rest, at least in part, on its exquisite ability to induce high levels of PKR in the cell and thereby to limit IFN- $\gamma$ mRNA translation through this negative feedback loop, blocking the excessive IFN- $\gamma$ gene expression that precedes clinical attacks.

Cell Research (2006) 16:148-153. doi:10.1038/sj.cr.7310020; published online 13 February 2006

Keywords: interferon- $\gamma$ mRNA, RNA pseudoknot, translational control, PKR, eIF2, multiple sclerosis, interferon- $\beta$

\section{Introduction}

The RNA-dependent protein kinase PKR is a major mediator of antiviral and inflammatory responses. This interferon (IFN)- and tumor necrosis factor (TNF)-inducible serine/threonine stress kinase is activated in the presence of double-stranded RNA (dsRNA), a hallmark of viral infection (reviewed in [1]). Once activated, PKR strongly inhibits translation by phosphorylating the $\alpha$-chain of eukaryotic initiation factor eIF2, that binds initiator methionyl tRNA in a GTP-dependent manner. Phospho-eIF2 $\alpha$ sequesters eIF2B, the GDP/GTP exchange factor that is limiting in the cell yet crucial for the recycling of eIF 2 between successive rounds of initiation; thus, phosphorylation of only $10-20 \%$ of eIF $2 \alpha$ suffices to block translation [2]. This renders translation is highly sensitive to inhibition by the activation of PKR. All cells express basal levels of PKR and use it to regulate their growth not only in response to

Correspondence: Raymond Kaempfer

Tel: +972-2-675-8389, Fax: +972-2-678-4010;

E-mail: kaempfer@hebrew.edu. stress but also under normal conditions.

PKR remains latent unless exposed to RNA, dsRNA being a powerful activator. Cells generally lack dsRNA but it is generated during the replication of RNA and DNA viruses. Activation of PKR by viral dsRNA leads to the shutdown of protein synthesis, resulting in a block of virus replication and selective apoptosis of the infected cell $[3,4]$. The antiviral action of IFNs and TNF is based to a major extent on their ability to induce PKR to high levels, enabling a prompt shutoff of translation when dsRNA appears after infection.

PKR contains tandem RNA-binding motifs. In the cell, most PKR is inactive because the second of these motifs interacts with the kinase domain, to mask the catalytic site. When a single dsRNA molecule binds to both motifs, a conformational change is induced in the kinase that allows dimerization and binding of ATP to the PKR dimer [5]. RNA duplexes of at least 11-13 bp are needed for kinase activation but their nucleotide sequence is not critical [6]. Once ATP is bound, the PKR dimer undergoes the transautophosphorylation necessary for its activation [7].

Natural regulators of PKR have been discovered recently 
A

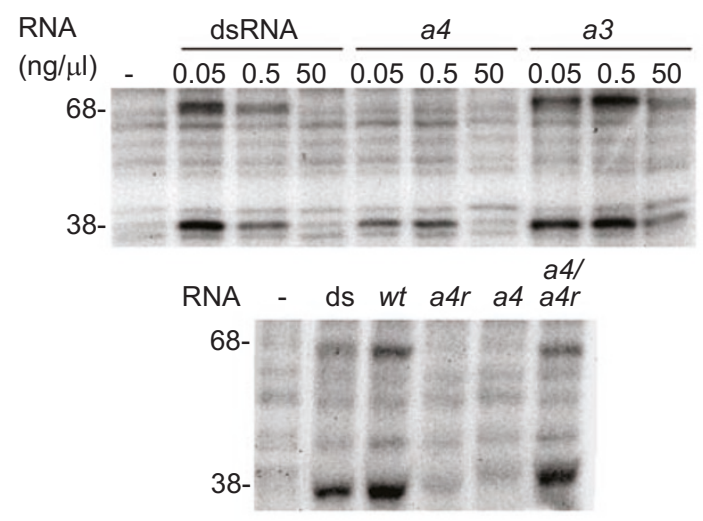

B

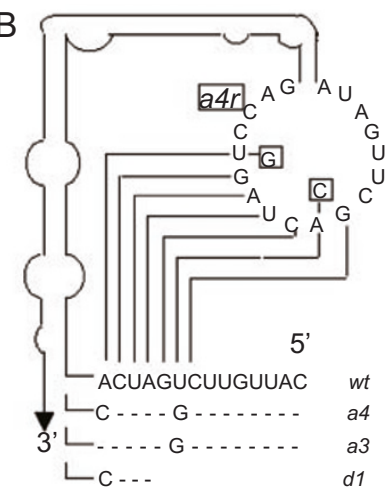

C

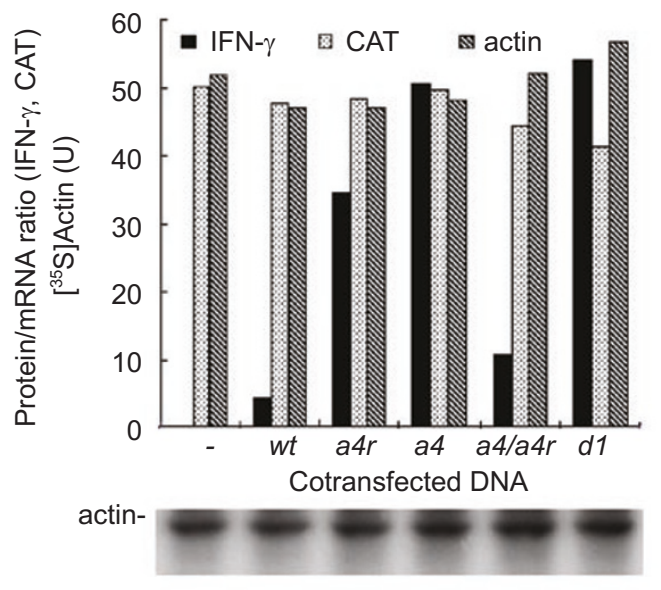

D
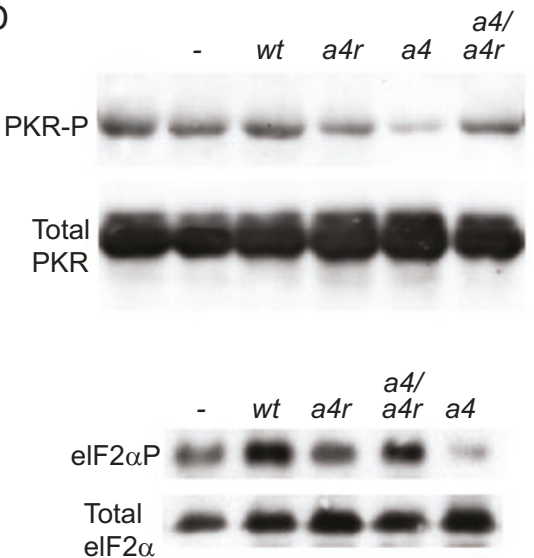

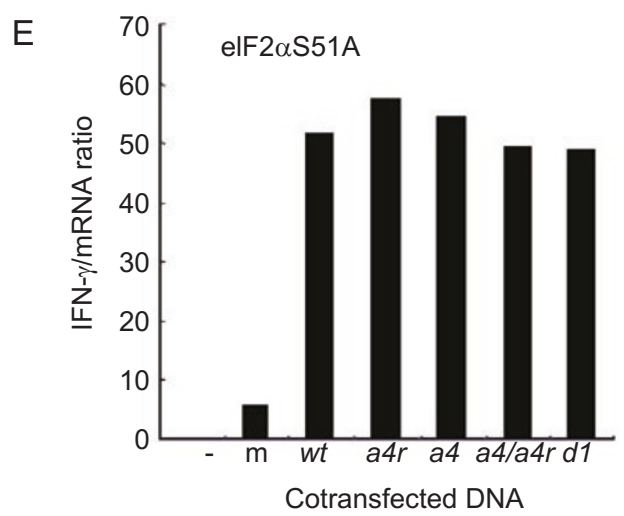

Figure 1 Human IFN- $\gamma$ mRNA locally activates PKR and regulates its translation through a pseudoknot in the 5'-UTR. (A) Activation of PKR by dsRNA, or by $a 4$ or $a 3$ mutant forms of the 5'-terminal 203-nt of IFN- $\gamma$ mRNA (203-nt RNA), assayed in the ribosome fraction of rabbit reticulocytes. Positions of PKR $(68 \mathrm{kDa})$ and eIF2 $\alpha(38 \mathrm{kDa})$ bands are shown. (B) Activation of PKR was assayed using wild type ( $w t$ ) or mutant 203-nt RNA or dsRNA (ds). Schematic on right shows 5'-terminal part of the IFN- $\gamma$ mRNA structure with mutational changes in the pseudoknot stem. (C) Translation efficiency was assayed in transfected BHK-21 cells, using $w t$ and mutant IFN- $\gamma$ cDNA as indicated, expressed under the myeloproliferative sarcoma virus promoter. IFN- $\gamma$ mRNA and CAT mRNA were quantitated by RNase protection analysis (not shown) and secreted IFN- $\gamma$ and intracellular CAT protein by ELISA. Relative translation efficiency is expressed as protein/mRNA ratio. Autoradiogram shows newly synthesized actin labeled with $\left[{ }^{35} \mathrm{~S}\right]$ methionine, assayed by immunoprecipitation; film intensity is plotted in arbitrary units. (D) Total and phosphorylated PKR and eIF2 $\alpha$ were assayed in cell lysates from (C) by immunoprecipitation and Western blotting with specific antibodies. (E) Translation efficiency was assayed in cells transfected as in (C), except that eIF2 $\alpha$ S51A was coexpressed. IFN- $\gamma$ and CAT mRNA were quantitated by RNase protection analysis (not shown) and IFN- $\gamma$ protein by ELISA. m, mock-transfected. From [1]. 
within the mRNAs that encode TNF- $\alpha$ and IFN- $\gamma$. These cis-acting RNA elements activate PKR even more potently than does dsRNA and function as sensors that respond to the level of PKR in the cell $[1,8,9]$. By locally activating PKR, these RNA sensors regulate TNF- $\alpha$ and IFN- $\gamma$ gene expression at the level of mRNA splicing and translation, respectively. TNF- $\alpha$ pre-mRNA and IFN- $\gamma$ mRNA mimic viral RNA as activators of PKR and may couple their regulated expression to the phosphorylation of eIF $2 \alpha$, a common outcome of different stress responses. Importantly, these novel RNA sensors respond to changes in intracellular levels of PKR that in turn fluctuate in response to the inflammatory cytokine microenvironment of the cell [9].

In multiple sclerosis, effective induction of PKR by IFN- $\beta$ may underlie the therapeutic efficacy of this cytokine. Elevated levels of PKR, locally activated by IFN $-\gamma$ mRNA, will cause attenuation of the excessive IFN- $\gamma$ production that is otherwise associated with the acute phases of this chronic autoimmune disease.

\section{Human IFN- $\gamma$ mRNA activates PKR through a 5'- terminal pseudoknot}

The human IFN- $\gamma$ gene uses a novel strategy to attenuate its own expression. Through a pseudoknot in its 5'-UTR, IFN- $\gamma$ mRNA activates PKR, resulting in the induction of eIF2 $\alpha$ phosphorylation and inhibition of its translation. This is the first example of an mRNA that regulates its own translation by activating PKR and the first case of PKR activation by an RNA pseudoknot [1].

IFN- $\gamma$ mRNA is a strong activator of PKR. This property is sensitive to mutations near the 5'-end of the RNA, which enhanced the translation of IFN- $\gamma$ mRNA in intact cells by up to thirtyfold. Knockout of the PKR gene or the introduction of any one of the PKR inhibitors, trans-dominant-negative mutant PKR, the vaccinia virus E3L protein, or 2 -aminopurine correspondingly enhance IFN- $\gamma$ mRNA translation [1].

In the $a 4$ mutant, that was severely impaired in its ability to activate PKR, formation of 2 out of 7 base pairs (bp) of the pseudoknot supported by structural analysis [1] was abolished, leaving only 4 contiguous bp (Figure 1B). In revertant $a 3$, base pairing was restored at one of these positions (Figure 1B). This single nucleotide change restored the ability to activate PKR (Figure 1A) to the level of $w t$ mRNA [1]. In the $d 1$ mutant, formation of the first 3 and the last bp of the proposed pseudoknot were eliminated, leading to essentially complete loss of the ability to activate PKR [1]. Thus, mutations in the first 14 nt of the 5'-UTR that reduce the ability of IFN- $\gamma$ mRNA to activate PKR impair the stability of the proposed pseudoknot. Maximal activation of PKR was seen when 7 contiguous bp were present in the stem $(w t)$, near-maximal activation with 5 bp (a3), severely reduced activation with $4 \mathrm{bp}(a 4)$ (Figure 1A), and only a weak residual activation with $3 \mathrm{bp}(d 1)$ [1].

To obtain direct evidence for the pseudoknot and to show that it is essential for activation of PKR by IFN- $\gamma$ mRNA, we introduced two point mutations in the apical loop where it shows complementarity to 5'-terminal nucleotides ( $a 4 r$; Figure 1B). If activation of PKR depends on the pseudoknot, then $a 4 r$ RNA should exhibit the phenotype of $a 4$ RNA, severely reduced ability to activate PKR, because the same two bp of the pseudoknot stem were abolished. When placed within $a 4$ mutant context, on the other hand, $a 4 r$ should restore a pseudoknot having a 7-bp stem. $a 4 r$ thus cause loss of function in $w t$ context yet gain of function in a4 context (a4/a4r).

These predictions were borne out (Figure 1B). Ability of $w t$ RNA to activate PKR was severely reduced by $a 4 r$, to the level seen for $a 4$. By contrast, ability to activate PKR was restored in full in a4/a4r RNA. This shows that the pseudoknot is essential for activation of PKR by IFN- $\gamma$ mRNA. Structure rather than sequence in the pseudoknot stem is important because activation of PKR was unaffected by compensatory changes in 2 out of $7 \mathrm{bp}$, in a 4/a4r RNA. This is the first example of activation of PKR by an RNA pseudoknot.

The effect of these mutations on translation efficiency of IFN- $\gamma$ mRNA in vivo was studied by transient expression of mRNA and protein in transfected cells (Figure 1C). Mutant $a 4$ and $d 1$ mRNA yielded high translation efficiency, 10 - and 12-fold over $w t$, respectively. Translation of $a 4 r$ mRNA was 7-fold greater than that of $w t$ mRNA. Whereas translation efficiency of IFN- $\gamma$ mRNA was enhanced by $a 4$ and $a 4 r$ mutations that impair the activation of PKR in vitro, it was reduced strongly by the compensatory a $4 / a 4 r$ double mutation which restores the potential for pseudoknot formation and activation of PKR. Translation efficiency in vivo (Figure 1C) thus is inversely proportional to the ability of these mRNAs to activate PKR in vitro (Figure 1B), providing evidence in vivo that the pseudoknot regulates translation of IFN- $\gamma$ mRNA. Translation efficiency of CAT mRNA and of endogenously expressed actin mRNA, on the other hand, was constant and independent of the nature of the co-expressed IFN- $\gamma$ mRNA (Figure 1C), showing that it is insensitive to IFN- $\gamma$ mRNA-mediated PKR activation. Apparently, activation of PKR by IFN- $\gamma$ mRNA is primarily local. The concept of local activation is further supported by our observation that in-frame joining of the 5 '-terminal 203 nt of IFN- $\gamma$ mRNA, which contain the entire PKR activator [1] and encode the IFN- $\gamma$ signal peptide and start of the mature protein, to the mature TNF- $\beta$ open reading frame resulted in translational repression of the chimeric TNF- $\beta$ mRNA through PKR activation. Thus, PKR-medi- 
ated autoregulation of translation was imposed on this recombinant mRNA, induced through the IFN- $\gamma$ element (S Namer and RK, unpublished results).

Phosphorylation of PKR and eIF2 $\alpha$, assayed in the transfected cell lysates, broadly reflected the pattern seen in vitro. Phosphorylation of both proteins was elevated over the basal level when $w t$ mRNA was expressed, below $w t$ level when $a 4$ or $a 4 r$ single mutant mRNA was expressed, and restored on expression of $a 4 / a 4 r$ double mutant mRNA (Figure 1D). $a 4 r$ mRNA showed higher residual phosphorylation activity in vivo than $a 4 \mathrm{mRNA}$ (Figure 1D), reflecting its somewhat lower translation efficiency (Figure 1C).

In principle, the inhibitory effect of PKR might result from binding of the kinase protein to the IFN- $\gamma$ 5'-UTR, thus rendering it as a translational repressor that prevents access of ribosomes to the AUG initiation codon. However, that is not the case. Co-expression of nonphosphorylatable S51A mutant eIF2 $\alpha$ was sufficient to restore the translation efficiency of $w t$ and a $4 / a 4 r$ mRNA to that of a4 and $d 1$ mutants that fail to activate PKR (Figure 1E), showing that it is the phosphorylation of eIF $2 \alpha$ by PKR that is critical for the pseudoknot-mediated control of IFN- $\gamma$ mRNA translation.

\section{A negative feedback loop in IFN- $\gamma$ mRNA transla- tion}

In IFN- $\gamma$ mRNA, a type $H$ pseudoknot stabilized by a stem of at least $5 \mathrm{bp}$ is essential for PKR activation (Figure 2A). Mutations that impair pseudoknot formation abolish the ability of IFN- $\gamma$ mRNA to activate PKR and greatly increase its translation, whereas compensatory mutations in the loop that restore pseudoknot structure activate PKR and induce translational repression (Figure 1). Deletion or mutation of the first $14 \mathrm{nt}$ of the IFN- $\gamma$ 5'-UTR during evolution would have sufficed to eliminate the pseudoknot (Figure 2A) and to enhance translation by as much as thirtyfold. Yet, the pseudoknot is phylogenetically conserved [1], attesting to its crucial role in reducing IFN- $\gamma$ mRNA translation to only a fraction of its full potential.

Our results imply a dynamic state in which the RNA sensor of PKR in IFN- $\gamma$ mRNA, able to activate PKR, is disrupted by the passage of ribosomes during translation and is refolded readily. The evolution of this sophisticated RNA structure points to its fundamental role as an RNA sensor of stress, through which the IFN- $\gamma$ mRNA molecule itself senses how much IFN- $\gamma$ was secreted into the cell's environment.

During the immune responses, as IFN- $\gamma$ and other inflammatory cytokines build up in the cell's microenvironment, they will induce higher levels of PKR and thus PKR will be activated more extensively by the RNA pseudoknot.
Through the resulting phosphorylation of eIF2 $\alpha$, a negative feedback loop is created and the production of IFN- $\gamma$ is progressively attenuated (Figure 2B) [9].

IFN- $\gamma$ is essential for protective immunity but when expressed in excess can induce autoimmune diseases such as SLE [10] or multiple sclerosis [11-13] and even toxic shock [14]. By contrast, overproduction of IFN- $\alpha$ or IFN- $\beta$ is less directly associated with pathology and a pseudoknot
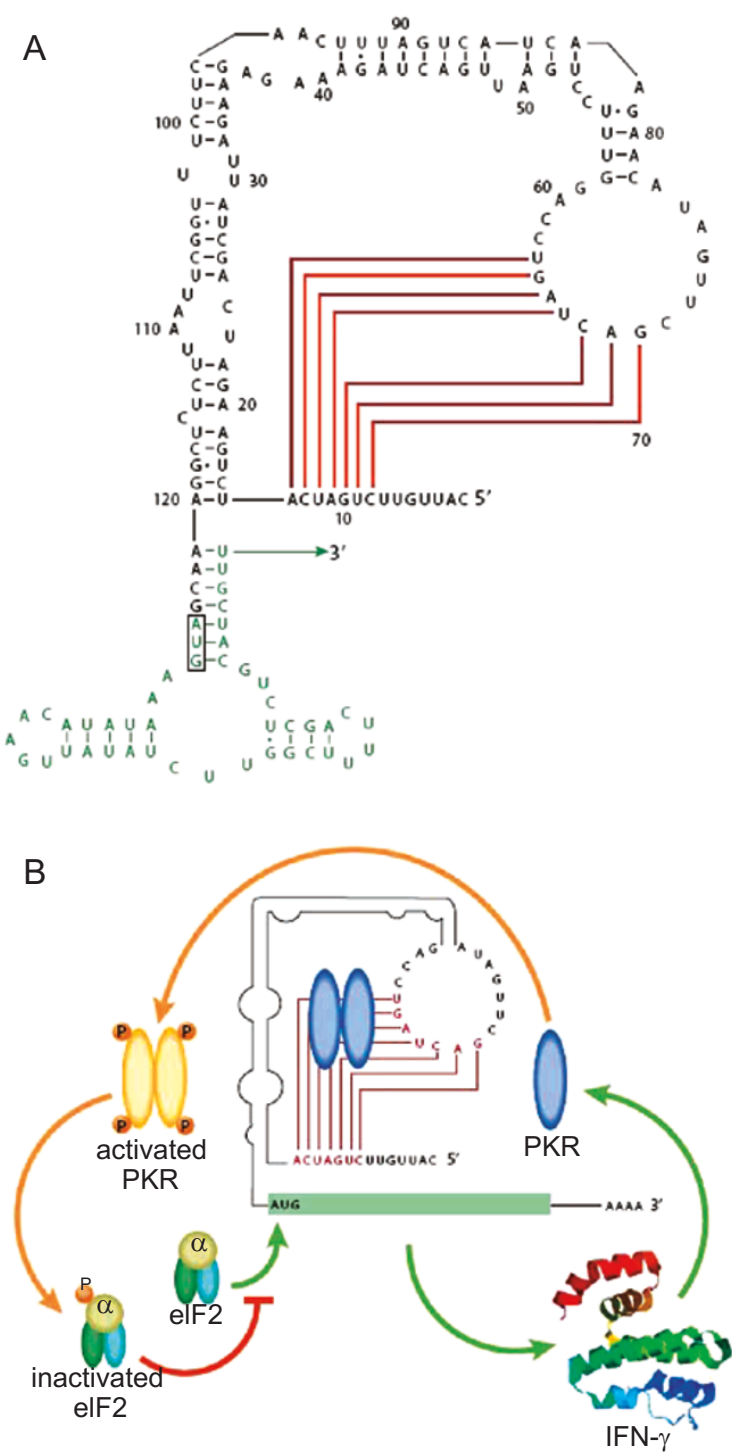

Figure 2 Human IFN- $\gamma$ mRNA activates PKR through a pseudoknot to inhibit its own translation. (A) Pseudoknot in the 5'-UTR of human IFN $-\gamma$ mRNA. Base pairing in the pseudoknot stem is shown in red and start of open reading frame in green. (B) Autoregulation of IFN- $\gamma$ synthesis. IFN- $\gamma$ mRNA is translated in dependence on eIF2, yielding IFN- $\gamma$ that can induce more PKR expression in the cell, creating a negative feedback loop. Inactive PKR binds into the pseudoknot, leading to a local activation of PKR. The resulting phosphorylation of eIF $2 \alpha$ blocks further translation of IFN- $\gamma$ mRNA. From [9]. 
motif that could mediate translational repression through PKR activation is not found in their 5'-UTRs.

The IFN- $\gamma$ pseudoknot activates PKR in the vicinity of IFN- $\gamma$ mRNA, giving rise to a localized inhibition of translation rather than a global effect. Translation of endogenous or foreign mRNA was insensitive to the nature of the IFN- $\gamma$ mRNA co-expressed in the cell, whether it was wild type or mutated in the pseudoknot (Figure 1). Hence, the pseudoknot in IFN- $\gamma$ mRNA is a cis-acting element that regulates only its own translation through the local activation of PKR in the vicinity of the mRNA molecule. The local nature of this activation rests most likely on the fact that rapid dephosphorylation of both PKR and its substrate eIF2 $\alpha$ is catalyzed by the ubiquitous protein phosphatase 1 (PP1), which acts to return PKR to an inactive state while restoring eIF $2 \alpha$ to full activity in initiation of translation. The local nature of PKR activation effected through the IFN- $\gamma$ mRNA pseudoknot element will enable the efficient creation of a negative feedback loop even when the mRNA is present at relatively low concentrations in the cytoplasm of the cell.

The crucial role of PP1 in maintaining translation in the cell is strongly supported by the fact that the anti-PKR strategy of Herpes simplex virus (HSV1) rests in large part on its induction of a virus-encoded regulatory subunit of PP1, $\gamma 34.5$, which activates the dephosphorylation process in the infected cell [15], allowing viral protein synthesis to proceed despite the activation of PKR elicited by viral replication.

\section{Multiple sclerosis treatment with IFN- $\beta$ : Blocking overexpression of IFN- $\gamma$}

It is now well established that multiple sclerosis patients benefit from IFN- $\beta$ treatment [16] but the molecular basis of this therapeutic effect is still unknown. Multiple sclerosis is an immune-mediated disease of the central nervous system white matter in which IFN- $\gamma$, a Th 1 cytokine, plays an important pathogenic role [11-13]. Injection of multiple sclerosis patients with recombinant IFN- $\gamma$ induced exacerbation of the disease [17] and an increase in IFN- $\gamma$ production by peripheral blood mononuclear cells precedes clinical attacks [18]. Furthermore, the inflammatory process in the brain of patients with multiple sclerosis is characterized by increased IFN- $\gamma$ expression [19].

Whereas IFN- $\gamma$ is a relatively weak inducer of PKR, the Type I interferons, especially IFN- $\beta$, are highly active inducers of PKR gene expression [4]. In the cell, the newly induced levels of PKR will be inactive unless they are exposed to activator RNA. However, the ability of IFN- $\gamma$ mRNA to activate PKR and thus to attenuate its own translation (Figure 2B) will become more pronounced when
PKR levels rise in the presence of IFN- $\beta$. Because activation of PKR is largely local (Figure 1), treatment with IFN$\beta$ will necessarily result in a selective inhibition of IFN- $\gamma$ mRNA translation. Accordingly, the negative feedback loop that is generated upon activation of IFN- $\beta$-enhanced levels of PKR, mediated by the pseudoknot in IFN- $\gamma$ mRNA, will lead to a blocking of excessive IFN- $\gamma$ production that is associated with acute flare-ups in multiple sclerosis.

Although it is not possible to demonstrate this molecular mechanism at the patient level, treatment of multiple sclerosis patients with IFN- $\beta$, which effectively reduces symptoms of disease, resulted in a significant decrease in circulating cells that produce IFN- $\gamma[20]$ and prevented the IFN- $\gamma$-induced disintegration of the endothelial junction integrity and barrier function [21]. These are but a few examples taken from a wider literature to support the view that the inhibition of IFN- $\gamma$ gene expression, induced by IFN- $\gamma$ mRNA-mediated activation of PKR resulting in translational attenuation of IFN- $\gamma$ production, as described here, is enhanced by IFN- $\beta$ treatment and thus may underlie its beneficial therapeutic effect.

\section{Acknowledgements}

Research in the author's laboratory was supported by grants from the Israel Science Foundation (537/03) and the Deutsche Forschungsgemeinschaft (HO-1116).

\section{References}

1 Ben-Asouli Y, Banai Y, Pel-Or Y, Shir A, Kaempfer R. Human interferon- $\gamma$ mRNA autoregulates its translation through a pseudoknot that activates the interferon-inducible protein kinase PKR. Cell 2002; 108:221-32.

2 Hinnebusch AG. Mechanism and regulation of initiator methionyl-tRNA binding to ribosomes. In: Sonenberg N, Hershey JWB, Mathews MB eds. Translational Control of Gene Expression. Cold Spring Harbor Laboratory Press: Cold Spring Harbor, New York 2000:185-243.

3 Meurs E, Chong K, Galabru J, et al. Molecular cloning and characterization of the human double-stranded RNA-activated protein kinase induced by interferon. Cell 1990; 62:379-90.

4 Stark GR, Kerr IM, Williams BR, Silverman RH, Schreiber RD. How cells respond to interferons. Annu Rev Biochem. 1998; 67:227-64.

5 Galabru J, Hovanessian A. Autophosphorylation of the protein kinase dependent on double-stranded RNA. J Biol Chem 1987; 262:15538-44.

6 Bevilacqua PC, Cech TR. Minor-groove recognition of doublestranded RNA by the double-stranded RNA-binding domain from the RNA-activated protein kinase PKR. Biochemistry 1996; 35:9983-94

7 Thomis DC, Samuel CE. Mechanism of interferon action: evidence for intermolecular autophosphorylation and autoactivation of the interferon-induced, RNA-dependent protein kinase PKR. 
J Virol 1993; 67:7695-700.

8 Osman F, Jarrous N, Ben-Asouli Y, Kaempfer R. A cis-acting element in the 3'-untranslated region of human TNF- $\alpha$ mRNA renders splicing dependent on the activation of protein kinase PKR. Genes Dev 1999; 13:3280-93.

9 Kaempfer R. RNA sensors: novel regulators of gene expression. EMBO Rep 2003; 4:1043-7.

10 Gerez L, Shkolnik T, Hirschmann O, et al. Hyperinducible expression of the interferon- $\gamma(\mathrm{IFN}-\gamma)$ gene and its suppression in systemic lupus erythematosus (SLE). Clin Exp Immunol 1997; 109:296-303.

11 Balashov KE, Olek MJ, Smith DR, Khoury SJ, Weiner HL. Seasonal variation of interferon- $\gamma$ production in progressive multiple sclerosis. Ann Neurol 1998; 44:824-8.

12 Karni A, Koldzic DN, Bharanidharan P, Khoury SJ, Weiner HL. IL-18 is linked to raised IFN- $\gamma$ in multiple sclerosis and is induced by activated $\mathrm{CD}^{+} \mathrm{T}$ cells via CD40-CD40 ligand interactions. J Neuroimmunol 2002; 125:134-40.

13 Sepulcre J, Sanchez-Ibarrola A, Moreno C, de Castro P. Association between peripheral IFN- $\gamma$ producing $\mathrm{CD} 8^{+} \mathrm{T}$-cells and disability score in relapsing-remitting multiple sclerosis. Cytokine 2005; 32:111-6.

14 Arad G, Levy R, Hillman D, Kaempfer R. Superantigen antagonist protects against lethal shock and defines a new domain for T-cell activation. Nat Med 2000; 6:414-21.
15 He B, Gross M, Roizman B. The $\gamma 34.5$ protein of herpes simplex virus 1 has the structural and functional attributes of a protein phosphatase 1 regulatory subunit and is present in a high molecular weight complex with the enzyme in infected cells. J Biol Chem 1998; 273:20737-43.

16 The IFNB Multiple Sclerosis Study Group. Interferon beta-1b is effective in relapsing-remitting multiple sclerosis. I. Clinical results of a multicenter, randomized, double-blind, placebo-controlled trial. Neurology 1993; 43:655-661.

17 Panitch HS, Hirsch RL, Haley AS, Johnson KP. Exacerbations of multiple sclerosis in patients treated with gamma interferon. Lancet 1987; 1:893-5.

18 Dettke M, Scheidt P, Prange H, Kirchner H. Correlation between interferon production and clinical disease activity in patients with multiple sclerosis. J Clin Immunol 1997; 17:293-300.

19 Woodroofe MN, Cuzner ML. Cytokine mRNA expression in inflammatory multiple sclerosis lesions: detection by non-radioactive in situ hybridization. Cytokine 1993; 5:583-8.

20 Furlan R, Bergami A, Lang R, et al. Interferon- $\beta$ treatment in multiple sclerosis patients decreases the number of circulating $\mathrm{T}$ cells producing interferon- $\gamma$ and interleukin-4. J Neuroimmunol 2000; 111:86-92.

21 Minagar A, Long A, Ma T, et al. Interferon (IFN)-beta 1a and IFN-beta $1 \mathrm{~b}$ block IFN- $\gamma$-induced disintegration of endothelial junction integrity and barrier. Endothelium 2003; 10:299-307. 